\title{
Connectedness versus diversification: two sides of the same coin
}

\author{
Maria-Laura Torrente ${ }^{1}$ (D) Pierpaolo Uberti ${ }^{1}$ (D)
}

Received: 7 August 2020 / Accepted: 5 January 2021 / Published online: 9 February 2021

(c) The Author(s) 2021

\begin{abstract}
In the financial framework, the concepts of connectedness and diversification have been introduced and developed respectively in the context of systemic risk and portfolio theory. In this paper we propose a theoretical approach to bring to light the relation between connectedness and diversification. Starting from the respective axiomatic definitions, we prove that a class of proper measures of connectedness verifies, after a suitable functional transformation, the axiomatic requirements for a measure of diversification. The core idea of the paper is that connectedness and diversification are so deeply related that it is possible to pass from one concept to the other. In order to exploit such correspondence, we introduce a function, depending on the classical notion of rank of a matrix, that transforms a suitable proper measure of connectedness in a measure of diversification. We point out general properties of the proposed transformation function and apply it to a selection of measures of connectedness, such as the well-known Variance Inflation Factor.
\end{abstract}

Keywords Diversification - Connectedness · Systemic risk · Portfolio risk

JEL codes $\mathrm{C} 60 \cdot \mathrm{D} 81 \cdot \mathrm{E} 30 \cdot \mathrm{G} 01 \cdot \mathrm{G} 10 \cdot \mathrm{G} 11$

\section{Introduction}

The idea that risk reduction for an investment portfolio can be achieved through a diversification approach is central in the Markowitz model, see Markowitz [19], and one of the principal reasons of its popularity. There is a wide consensus on the risk reduction properties of the diversification, both from practitioners and academics. The qualitative definition of diversification is natural for portfolio managers: an investment portfolio is well diversified when it is not exposed to individual shocks occurring to its constituents. The formal definition of diversification remains an elusive concept and it is hardly made explicit in portfolio

Maria-Laura Torrente

marialura.torrente@economia.unige.it

Pierpaolo Uberti

uberti@economia.unige.it

1 Dipartimento di Economia, Università di Genova, Via Vivaldi 5, 16126 Genoa, Italy 
optimization studies, with an exception for the paper by Meucci [20] and related studies. In its paper, Meucci proposes to use principal component analysis to extract uncorrelated risk factors starting from the underlying assets.

The vagueness of its definition gave rise to a huge literature focusing on different aspects of diversification. Many different approaches for diversification based portfolio strategies have been studied in the literature: among the others, we recall the naive diversification strategy and the measurement of its out of sample performance, see DeMiguel et al. [7], the equally risk contribution diversification strategy, see Roncalli and Weisang [23] and Qian [22], the application of the approach proposed by Meucci, see Lohre et al. [17], the determination of maximum diversification portfolios, see Choueifaty and Coignard [5], the investment strategy based on the maximization of the diversification ratio, see Choueifaty et al. [6], the strategy based on the generalized Rao's entropy used as a diversification measure, see Choueifaty et al. [6]. Moreover, the literature presents further approaches that relate the diversification to different aspects of portfolio composition, like sector diversification and geographical diversification, see for example Hauser and Vermeersch [14] and Diamond and Abdullah [8], or product based diversification, see for example Bernardi et al. [1], for the diversification approach based on commodities risk factors. In this paper we refer to the axiomatic definition of coherent diversification measures proposed by Koumou and Dionne [16].

While the idea of diversification approximately dates back to 1950s, the concept of connectedness is relatively new. It has been first introduced by Diebold and Yilmaz, see Diebold and Yilmaz [9], and successively developed in subsequent papers, see among the others Diebold and Yilmaz [10] and Diebold and Yilmaz [11], in which the authors well describe the concept of connectedness, pointing out the lack of a formal definition and the resulting vagueness of the notion. Recently, an attempt to formalize the concept of connectedness through an axiomatic approach has been proposed in Maggi et al. [18]. The idea behind connectedness is to measure the degree of the inter-relations and the inter-dependencies between the components of a whole system and to summarize the information in a single number. Despite its potential generality, restricting to the financial context, a high connectedness reflects a strong interdependence between the elements of the system. This interpretation highlights the intuitive relation between connectedness and systemic risk. For a comprehensive review of the measures proposed in the literature for systemic risk analysis we refer to the survey by Bisias et al. [4].

In the context of portfolio theory, diversification is usually achieved through the computation of the optimal weights to invest in. On the opposite, connectedness is not directly related to portfolio theory and portfolio weights are not considered when evaluating the connectedness of a market or an economic system or even the assets of a portfolio. Despite the differences and the distinctive features among connectedness and diversification, in this paper we aim at investigate and highlight the deep relation between the two concepts.

An important similarity can be found in the role of correlation. As pointed out in Diebold and Yilmaz [11], in the literature on connectedness the use of correlation-based measures is widespread and the concept of connectedness may be seen as a generalization of the concept of correlation while, on the other hand, correlation is the standard base for the evaluation of diversification. One further link between connectedness and diversification can be found in the role of the eigenvalues in both fields, see for instance Meucci [20] and Maggi et al. [18], where the eigenvalues are used to evaluate both diversification and connectedness.

In accordance to standard portfolio theory asserting that the portfolio risk can be decomposed into its systematic and idiosyncratic components, diversification is geared at eliminating the idiosyncratic risk while connectedness estimates the systemic risk component. In this framework, the relation between the two concepts is immediate and connectedness 
can be thought as the complement of the diversification with respect to the the total risk of a portfolio. Referring to the idiosyncratic and systemic components of the risk, it is useful to interpret the monotonicity properties of the two measures with respect to the size of the portfolio; in particular, diversification improves with large portfolios while connectedness, representing the systemic component, is not positively affected by a growing number of assets. We will show that a high level of connectedness corresponds to a reduced possibility to build a well diversified portfolio. On the opposite, the possibility to build a well diversified portfolio is related to a low level of connectedness between the assets, i.e. the assets provide good opportunities of diversification.

In this paper, in order to highlight the similarities between connectedness and diversification, we explicitly introduce the weights in the computation of connectedness, generalizing its definition and showing that standard measures of connectedness can be related to the special case of the equally weighted portfolios. Further, we effectively analyze this correspondence by comparing the axiomatic frameworks of the Proper Measures of Connectedness (PMCs) and the Coherent Portfolio Diversification Measures (CPDMs), see Maggi et al. [18] and Koumou and Dionne [16] respectively. We note that, due to the peculiarities of the two settings, the requirements for PMCs are less restrictive than the ones for CPDMs. As a consequence, a specific PMC needs to verify some additional properties to be properly related to a CPDM.

Our approach is general. We introduce a suitable function $\mathcal{F}$ and prove that, if applied to a subclass of PMCs verifying given additional properties, such as, for example, quasi-convexity and homogeneity, it transforms such measures into CPDMs. Then, we consider a selection of four PMCs, the Maximum Variance Inflation Factor (M-VIF), see Belsey et al. [2], the Power Mean measures $\mu_{k}^{1}$, see Maggi et al. [18], the Market Rank Indicator, see Figini et al. [12] and the Cumulative Risk Fraction $h_{k}$, see Billio et al. [3], and we apply $\mathcal{F}$ defining four original diversification measures. We prove in details that $\mathcal{F}_{\mathrm{M}-\mathrm{VIF}}$ is a CPDM and show that the measures of diversification $\mathcal{F}_{\mu_{k}^{1}}, \mathcal{F}_{s_{k}}$ and $\mathcal{F}_{h_{k}}$ partially verify the theoretical requirements of CPDMs.

The paper is organized as follows: in Sect. 2 we recall the axiomatic frameworks of PMCs and CPDMs; in Sect. 3 we introduce the function $\mathcal{F}$ providing the relationship between the concepts of connectedness and diversification, dwelling on the additional required properties on PMCs necessary to construct a CPDM; in Sect. 4 we apply $\mathcal{F}$ to a selection of PMCs and prove the properties they verify as induced measures of diversification; finally, in Sect. 5 we discuss the proposed results.

\section{Connectedness and diversification: axiomatic definitions}

In this section we recall the general notions of Proper Measures of Connectedness (PMCs) and Coherent Diversification Measures (CDMs) briefly specifying the economic interpretation of the theoretical properties required for the axiomatic definition of the two families of measures; for a more detailed description we refer to Maggi et al. [18] and Koumou and Dionne [16] respectively. In order to compare the measures of connectedness and diversification in a meaningful perspective, we refer to the portfolio allocation scheme, where the CPDMs have been principally developed. 


\subsection{Notation and preliminaries}

Let $m \geq n \geq 2$, let Mat $_{m \times n}$ be the set of $m \times n$ real matrices and $\mathcal{M}_{m \times n}$ be the subset of Mat $_{m \times n}$ containing all the full-rank matrices, i.e. $\operatorname{rank}(A)=n, \forall A \in \mathcal{M}_{m \times n}$. We indicate with $\mathbf{0}_{m \times n}$ and $\mathbf{1}_{m \times n}$ the $m \times n$ matrices whose elements are all equal to zero and one respectively. For brevity, we will also use the simpler notation $\mathbf{1}_{n}$, or even $\mathbf{1}$ if the size of the vector is clear from the context, to denote the vector $\mathbf{1}_{n \times 1}$. Throughout the paper, we will interpret the entries of the column vectors $A^{j}$, with $j=1, \ldots, n$, of any matrix $A=\left(a_{i j}\right)$ as the historical observations of the $j$ th return of the portfolio, so that the $m \times n$ matrix $A$ is the usual matrix of portfolio returns with $m$ historical data and $n$ assets. We denote by $\overline{A^{j}}$ the mean of $A^{j}$ and let $\bar{A}=\left(\overline{A^{1}} \mathbf{1}_{m \times 1}, \ldots, \overline{A^{n}} \mathbf{1}_{m \times 1}\right) ; \bar{A}$ contains the averages of the historical returns of the assets in the portfolio. We let $A^{t}$ be the transposed of $A$ and $\sigma_{1}(A) \geq \ldots \geq \sigma_{r}(A)>0$, with $r=\operatorname{rank}(A)$, be the singular values of $A$ listed as usual in nonincreasing order; further, for any $\alpha \in \mathbb{R}$, we denote by $A+\alpha$ and $\alpha A$ the $m \times n$ matrices whose $(i, j)$ entry is $a_{i j}+\alpha$ and $\alpha a_{i j}$, respectively. We let $P=\left(p_{1}, \ldots, p_{r}\right)$ be the list of pivots obtained by the classical Gauss-Jordan elimination applied to $A$ and $A^{P}=\left(A^{p_{1}}, \ldots, A^{p_{r}}\right) \in \mathcal{M}_{m \times r}$. We denote by $\rho(\cdot, \cdot)$ the Pearson correlation coefficient, by $\langle\cdot, \cdot\rangle$ the standard scalar product and by $\|\cdot\|$ the Euclidean norm of $\mathbb{R}^{n}$. Finally, we let $\mathbb{W}_{n}=\left\{\boldsymbol{w}=\left(w_{1}, \ldots, w_{n}\right)^{t} \in \mathbb{R}_{\geq 0}^{n} \mid \sum_{j=1}^{n} w_{j}=1\right\}$ be the set of long-only portfolios associated to $A$, so that $w_{j}$ is the weight of asset $A^{j}$ in the portfolio $\boldsymbol{w}$, and we define $W=\operatorname{diag}(\boldsymbol{w}) \in \operatorname{Mat}_{n \times n}$ as the diagonal matrix with elements $w_{1}, \ldots, w_{n}$. A portfolio made up of a single asset $j$ is denoted by $\boldsymbol{e}_{j}^{t}$, where $\boldsymbol{e}_{1}, \ldots, \boldsymbol{e}_{n}$ is the standard basis of $\mathbb{R}^{n}$.

We denote by $\mathcal{H}^{m, n}$ the set of all positive real-valued functions defined over the set of matrices $\mathrm{Mat}_{m \times n} \backslash\left\{\boldsymbol{0}_{m \times n}\right\}$ and by $\mathcal{H}_{\boldsymbol{w}}^{m, n}$ the set of all positive real-valued functions defined over $\mathbb{W}_{n} \times \operatorname{Mat}_{m \times n} \backslash\left\{\boldsymbol{0}_{m \times n}\right\}$. In the following definition we list some properties of functions belonging to $\mathcal{H}^{m, n}$.

Definition 1 Let $g \in \mathcal{H}^{m, n}$; then $g$ is said to be

(i) invariant under independent translations if $g(A+B)=g(A)$ for each nonzero $A \in$ $\mathrm{Mat}_{m \times n}$ having no rows and no columns equal to $\mathbf{1}_{1 \times n}$ and $\mathbf{1}_{m \times 1}$ respectively, and $B=\left(b_{i j}\right) \in$ Mat $_{m \times n}$ such that $b_{i j}=\beta_{j} \in \mathbb{R}$ for each $i=1, \ldots, m$ and $j=1, \ldots, n$;

(ii) invariant for size if $g(A \mid b)=g(A)$ for each nonzero $A \in \operatorname{Mat}_{m \times n}$ and $b \in \operatorname{Mat}_{m \times 1}$ such that $\operatorname{rank}(A \mid b)=\operatorname{rank}(A)$;

(iii) invariant under columns permutations if $g(A \Pi)=g(A)$ for each $A \in \mathcal{M}_{m \times n}$ and for any permutation matrix $\Pi \in$ Mat $_{n \times n}$;

(iv) invariant under one scalar multiplication if $g\left(A^{1}, \ldots, \alpha A^{k}, \ldots, A^{n}\right)=g(A)$ for each nonzero $A \in \operatorname{Mat}_{m \times n}, \alpha>0$ and $k \in\{1, \ldots, n\}$;

(v) homogeneous of degree 0 if $g(\alpha A)=g(A)$ for each nonzero $A \in \operatorname{Mat}_{m \times n}$ and $\alpha>0$;

(vi) quasi-convex if $g(\alpha A+(1-\alpha) B) \leq \max \{g(A), g(B)\}$ for each $A, B \in \mathcal{M}_{m \times n}$ and $\alpha \in[0,1]$.

Remark 1 It is immediate to observe that the invariance under one scalar multiplication is a very strong property which implies that the function is invariant under (even different) simultaneous scalar multiplication of each column. Further, in particular, it implies the degree 0 homogeneity property. 


\subsection{Connectedness}

Definition 2 (PMCs) A real-valued function $C: \mathcal{M}_{m \times n} \rightarrow \mathbb{R}$, defined by $C(A)$ for each $A \in \mathcal{M}_{m \times n}$ is a Proper Measure of Connectedness (PMC) if it satisfies the following minimal properties $C 1, C 2, C 3$ and $C 4$.

C1. $C(A) \geq 0$, for any $A \in \mathcal{M}_{m \times n}$.

C2. $C(A)$ is invariant for any permutation of the columns of $A \in \mathcal{M}_{m \times n}$.

C3. $C(A)>C(B)$ if and only if $C(\alpha A)>C(\alpha B)$, for any $A, B \in \mathcal{M}_{m \times n}$ and $\alpha>0$.

$C 4$. Let $a_{1}, a_{2}, a_{3} \in \mathcal{M}_{m \times 1}$, with $\left\|a_{2}\right\|=\left\|a_{3}\right\|$ and $\left\langle\mathbf{1}, a_{2}\right\rangle=\left\langle\mathbf{1}, a_{3}\right\rangle=0$. If $\left|\rho\left(a_{1}, a_{2}\right)\right| \geq$ $\left|\rho\left(a_{1}, a_{3}\right)\right|$ then $C\left(\left(a_{1}, a_{2}\right)\right) \geq C\left(\left(a_{1}, a_{3}\right)\right)$ where, with a small abuse of notation, we assume that $\left(a_{1}, a_{2}\right),\left(a_{1}, a_{3}\right) \in \mathcal{M}_{m \times 2}$.

It is useful to observe that if $C$ satisfies property (v) of Definition 1 , then $C$ satisfies property $C 3$.

In the financial context, the minimal properties required in Definition 2 for a PMC are intuitive. If we exclude property $C 1$ that is a pure technical condition, a PMC is not affected by the order the assets are considered, see property $C 2$. Property $C 3$ requires that a positive rescaling in the data does not significantly impact the structure of connectedness. Finally, with property $C 4$, a PMC is required to embody at least the information of the correlation structure of the data so that a higher correlation results in a higher connectedness. The opposite implication does not hold; an increase in the value of connectedness could depend on different causes than an increase in the value of correlation among the data.

Definition 2 provides the general notion of PMCs, as given in (Maggi et al., [18]). In this paper, in order to use PMCs in the context of standard portfolio theory, we will consider connectedness measures defined on matrices $A W$, where $W=\operatorname{diag}(\boldsymbol{w})$ is the diagonal matrix associated to a tuple of portfolio weights $w \in \mathbb{W}_{n}$. In this way, Definition 2 boils down to the standard definition of PMCs when the function $C$ is homogeneous of degree 0 and the considered portfolio is $\frac{1}{n} \mathbf{1}$, that is the equally weighted portfolio.

\subsection{Diversification}

In the following definition we recall the Coherent Portfolio Diversification Measures (CPDMs).

Definition 3 (CPDMs) A real-valued function $\Phi^{(m, n)}: \mathbb{W}_{n} \times$ Mat $_{m \times n} \rightarrow \mathbb{R}$, simply denoted by $\Phi$ when no confusion arises, is a Coherent Portfolio Diversification Measure (CPDM) if it satisfies the following axioms $D 1, D 2, D 3, D 4, D 5, D 6, D 7, D 8$ and $D 9$.

D1. (Quasi-concavity) For any $\boldsymbol{w}_{1}, \boldsymbol{w}_{2} \in \mathbb{W}_{n}$ and $\alpha \in[0,1]$ it holds that $\Phi\left(\alpha \boldsymbol{w}_{1}+(1-\right.$ $\left.\alpha) \boldsymbol{w}_{2}, A\right) \geq \min \left\{\Phi\left(\boldsymbol{w}_{1}, A\right), \Phi\left(\boldsymbol{w}_{2}, A\right)\right\}$, with strict inequality for at least one value of $\alpha$.

D2. (Size degeneracy) There is a constant $\underline{\Phi} \in \mathbb{R}$ such that $\Phi\left(\boldsymbol{e}_{j}, A\right)=\underline{\Phi}$, for each $j=1, \ldots, n$.

D3. (Risk degeneracy) Let $A$ be such that $A^{i}=A^{j}$ for each $i, j=1, \ldots, n$; then, there is a constant $\underline{\Phi} \in \mathbb{R}$ such that $\Phi(\boldsymbol{w}, A)=\underline{\Phi}$ for each $\boldsymbol{w} \in \mathbb{W}_{n}$.

D4. (Reverse risk degeneracy) For any $\boldsymbol{w} \in \mathbb{W}_{n}$ such that $\boldsymbol{w} \neq \boldsymbol{e}_{j}, j=1, \ldots, n$, and $w_{i}>0$, $i=1, \ldots, n$, we consider the equation $\Phi(\boldsymbol{w}, A)=\underline{\Phi}$ in the variable $A \in \operatorname{Mat}_{m \times n}$ and assume that a solution $A^{*}$ exists. Then $A^{*}$ is lower comonotonic. 
D5. (Duplication invariance) Let $k \in\{1, \ldots, n\}$ and $A^{+}=\left(A \mid A^{k}\right) \in \operatorname{Mat}_{m \times(n+1)}$. Then $\Phi\left(\boldsymbol{w}^{+}, A^{+}\right)=\Phi(\boldsymbol{w}, A)$ for each $\boldsymbol{w} \in \mathbb{W}_{n}$ and $\boldsymbol{w}^{+} \in \mathbb{W}_{n+1}$ such that $w_{j}^{+}=w_{j}$, for each $j=1, \ldots, n, j \neq k$, and $w_{k}^{+}+w_{n+1}^{+}=w_{k}$.

D6. (Size monotonicity) Let $A^{+}=\left(A \mid A^{n+1}\right) \in \operatorname{Mat}_{m \times(n+1)}$, with $A^{n+1} \neq A^{j}$ for each $j=1, \ldots, n$. Then $\Phi\left(\boldsymbol{w}^{+}, A^{+}\right) \geq \Phi(\boldsymbol{w}, A)$ for each $\boldsymbol{w} \in \mathbb{W}_{n}$ and $\boldsymbol{w}^{+} \in \mathbb{W}_{n+1}$ such that $\left(w_{1}^{+}, \ldots, w_{n}^{+}\right)$is proportional to $w$.

D7. (Translation invariance) Let $\alpha \in \mathbb{R}$; then $\Phi(\boldsymbol{w}, A+\alpha)=\Phi(\boldsymbol{w}, A)$ for each $\boldsymbol{w} \in \mathbb{W}_{n}$.

D8. (Homogeneity) Let $\alpha \geq 0$; then there exists $r \in \mathbb{R}$ such that $\Phi(\boldsymbol{w}, \alpha A)=\alpha^{r} \Phi(\boldsymbol{w}, A)$ for each $w \in \mathbb{W}_{n}$.

D9. (Symmetry) Let $\Pi \in$ Mat $_{n \times n}$ be a permutation matrix; then $\Phi(\boldsymbol{w} \Pi, A \Pi)=\Phi(\boldsymbol{w}, A)$ for each $\boldsymbol{w} \in \mathbb{W}_{n}$.

In Koumou and Dionne [16], axiom D1 requires quasi-convexity or, alternatively, convexity. Considering that convexity implies quasi-convexity, we preferred to directly require quasi-convexity.

In the financial context, the minimal properties for a CPDM listed in Definition 3 have the following economic interpretation. The preference towards diversification such that holding different assets increases diversification is translated by property $D 1$. Property $D 2$, size degeneracy, reflects that single asset portfolios are equivalent to the minimum level of diversification. Properties $D 3$ and $D 4$, risk degeneracy and reverse risk degeneracy, respectively state that including perfect similar assets in a portfolio do not help diversification and that a portfolio composed by assets with independent returns and single asset portfolios do not have the same level of diversification. Property D5, duplication invariance, is required to avoid that diversification measurement is affected by multiple representative assets. Property $D 6$, size monotonicity, reflects the relation between portfolio diversification and the number of assets in the portfolio. Adding a deterministic cash return does not impact portfolio diversification: this idea is described by property $D 7$. Diversification is not affected by scalar transformations of the input data, see property $D 8$ about homogeneity. Finally, as in the context of connectedness, diversification is required to be independent from any permutation of the assets in a portfolio, see property $D 9$ about symmetry.

\section{From connectedness to diversification}

In this section we propose a method to construct original portfolio diversification measures starting from given PMCs. In order to explicitly define a subclass of CPDMs we need to require the PMCs to verify some extra properties, restricting the class of PMCs that are of potential interest. The passage from connectedness to diversification is possible through the use of a specific transformation function $\mathcal{F}: \mathcal{H}^{m, n} \rightarrow \mathcal{H}_{\boldsymbol{w}}^{m, n}$ defined as follows.

Definition 4 (Transformation function) Let $C \in \mathcal{H}^{m, n}$ and $f_{C} \in \mathcal{H}_{w}^{m, n}$ be the function defined by:

$$
f_{C}(\boldsymbol{w}, A)=\operatorname{rank}(A W)+\frac{1}{C(A W)}-1
$$

for each $\boldsymbol{w} \in \mathbb{W}_{n}$ and $A \in \operatorname{Mat}_{m \times n} \backslash\left\{\boldsymbol{0}_{m \times n}\right\}$, where $W=\operatorname{diag}(\boldsymbol{w}) \in \operatorname{Mat}_{n \times n}$.

The transformation function $\mathcal{F}: \mathcal{H}^{m, n} \rightarrow \mathcal{H}_{w}^{m, n}$ is defined by $\mathcal{F}(C)=\mathcal{F}_{C} \in \mathcal{H}_{w}^{m, n}$, for each $C \in \mathcal{H}^{m, n}$, where $\mathcal{F}_{C}$ acts on $\mathbb{W}_{n} \times \operatorname{Mat}_{m \times n} \backslash\left\{\mathbf{0}_{m \times n}\right\}$ as follows:

$$
\mathcal{F}_{C}(\boldsymbol{w}, A)=1-\frac{1}{f_{C}(\boldsymbol{w}, A)}
$$


for each $\boldsymbol{w} \in \mathbb{W}_{n}$ and $A \in \operatorname{Mat}_{m \times n} \backslash\left\{\mathbf{0}_{m \times n}\right\}$.

Note that in the rest of the paper we will only consider the nontrivial and meaningful case $\operatorname{rank}(A W)>0$. Intuitively, the behaviour of $\mathcal{F}_{C}$ agrees with the distinguishing feature of diversification: indeed, if we consider the simplest case of the equally weighted portfolios, $\mathcal{F}_{C}$ decreases as the function $C$ increases, which highlights the main relationship between the notions of diversification and connectedness (if the overall connectedness between the assets increases, the diversification opportunities when creating a portfolio with these assets are expected to reduce).

The following proposition gathers some basic properties on $\mathcal{F}_{C}$.

Proposition 1 Let $C$ be a function of $\mathcal{H}^{m, n}$ that assumes values in $[1,+\infty)$. For each nonzero $A, A_{1}, A_{2} \in \operatorname{Mat}_{m \times n}$ and $\boldsymbol{w}, \boldsymbol{w}_{1}, \boldsymbol{w}_{2} \in \mathbb{W}_{n}$, with $W=\operatorname{diag}(\boldsymbol{w}), W_{1}=\operatorname{diag}\left(\boldsymbol{w}_{1}\right), W_{2}=$ $\operatorname{diag}\left(\boldsymbol{w}_{2}\right) \in \operatorname{Mat}_{n \times n}$, the following properties hold.

1. $\mathcal{F}_{C}(\boldsymbol{w}, A)=0$ if and only if $\operatorname{rank}(A W)=1($ and $C(A W)=1)$;

2. $\mathcal{F}_{C}(\boldsymbol{w}, A) \in[0,1)$;

3. $1-\frac{1}{\operatorname{rank}(A W)-1}<\mathcal{F}_{C}(\boldsymbol{w}, A) \leq 1-\frac{1}{\operatorname{rank}(A W)}$;

4. if $\operatorname{rank}\left(A_{1} W_{1}\right)<\operatorname{rank}\left(A_{2} W_{2}\right)$ then $\mathcal{F}_{C}\left(\boldsymbol{w}_{1}, A_{1}\right)<\mathcal{F}_{C}\left(\boldsymbol{w}_{2}, A_{2}\right)$;

5. if $w_{j}>0$, for each $j=1, \ldots, n$, then $A^{*}$ is a solution of the equation $\mathcal{F}_{C}(\boldsymbol{w}, A)=0$ if and only if $\operatorname{rank}\left(A^{*}\right)=1$.

Proof 1 . The condition $\mathcal{F}_{C}(\boldsymbol{w}, A)=0$ is equivalent to the equality $f_{C}(\boldsymbol{w}, A)=$ $\operatorname{rank}(A W)+\frac{1}{C(A W)}-1=1$; using the assumptions on the function $C$ we have

$$
\operatorname{rank}(A W)-1<f_{C}(\boldsymbol{w}, A) \leq \operatorname{rank}(A W),
$$

which yields $\operatorname{rank}(A W)=1$ and consequently $C(A W)=1$. On the other hand, imposing the positivity of the function $\mathcal{F}_{C}$, the condition $\operatorname{rank}(A W)=1$ implies that $C(A W)=1$, and so $\mathcal{F}_{C}(\boldsymbol{w}, A)=0$, so item 1 is proved.

2. From now on, we consider the case $\operatorname{rank}(A W) \geq 2$. Following the hypothesis on the function $C$ we have $f_{C}(\boldsymbol{w}, A)>1$, therefore item 2 is proved.

3. Using inequality (1) and Definition 4 , item 3 immediately follows.

4. The hypothesis of item 4 yields $\operatorname{rank}\left(A_{1} W_{1}\right) \leq \operatorname{rank}\left(A_{2} W_{2}\right)-1$ so, using item 3 , we have:

$$
\mathcal{F}_{C}\left(\boldsymbol{w}_{1}, A_{1}\right) \leq 1-\frac{1}{\operatorname{rank}\left(A_{1} W_{1}\right)} \leq 1-\frac{1}{\operatorname{rank}\left(A_{2} W_{2}\right)-1}<\mathcal{F}_{C}\left(\boldsymbol{w}_{2}, A_{2}\right),
$$

therefore item 4 is proved.

5. From item 1 , any solution $A^{*}$ of the equation $\mathcal{F}_{C}(\boldsymbol{w}, A)=0$ satisfies $\operatorname{rank}\left(A^{*} W\right)=1$. Since, by hypothesis, $\operatorname{rank}(W)=n$, Sylvester inequality, see (Horn and Johnson, [15, Section 0.4.5]), yields $1=\operatorname{rank}\left(A^{*} W\right) \geq \operatorname{rank}\left(A^{*}\right)+n-n=\operatorname{rank}\left(A^{*}\right)$, therefore item 5 is proved.

We now provide a deeper characterization of $\mathcal{F}_{C}$ on the base of additional properties required for $C$. To this end, we need to introduce the following technical lemma yielding useful features of the rank operator in order to investigate the concavity properties of $\mathcal{F}_{C}$.

Lemma 1 Let $A \in \mathcal{M}_{m \times n}$ and $\boldsymbol{w}_{1}, \boldsymbol{w}_{2} \in \mathbb{W}_{n}$, with $W_{1}=\operatorname{diag}\left(\boldsymbol{w}_{1}\right), W_{2}=\operatorname{diag}\left(\boldsymbol{w}_{2}\right) \in$ $\mathrm{Mat}_{n \times n}$. For any $\alpha \in(0,1)$, the following result holds:

$$
\operatorname{rank}\left(\alpha A W_{1}+(1-\alpha) A W_{2}\right) \geq \max \left\{\operatorname{rank}\left(A W_{1}\right), \operatorname{rank}\left(A W_{2}\right)\right\},
$$


with strict inequality if and only if $I_{k} \subsetneq I_{1} \cup I_{2}, k=1,2$, where $I_{k}=\left\{j \in\{1, \ldots, n\} \mid w_{k j}>\right.$ $0\}$.

Proof Let $W_{\alpha}=\alpha W_{1}+(1-\alpha) W_{2} \in \mathrm{Mat}_{m \times n}$ and $A W_{\alpha}=\alpha A W_{1}+(1-\alpha) A W_{2}$. Since $\operatorname{rank}(A)=n$, Sylvester inequality, see Horn and Johnson [15, Section 0.4.5], yields the following equalities

$$
\operatorname{rank}\left(A W_{\alpha}\right)=\operatorname{rank}\left(W_{\alpha}\right),
$$

$\operatorname{rank}\left(A W_{1}\right)=\operatorname{rank}\left(W_{1}\right)$ and $\operatorname{rank}\left(A W_{2}\right)=\operatorname{rank}\left(W_{2}\right)$. Further, $W_{\alpha}$ is a diagonal matrix whose entries are the strictly convex combinations of the corresponding elements of $W_{1}$ and $W_{2}$, so that its strictly positive entries are indexed by $I_{1} \cup I_{2}$. It easily follows that

$$
\operatorname{rank}\left(W_{\alpha}\right) \geq \max \left\{\operatorname{rank}\left(W_{1}\right), \operatorname{rank}\left(W_{2}\right)\right\}=\max \left\{\operatorname{rank}\left(A W_{1}\right), \operatorname{rank}\left(A W_{2}\right)\right\}
$$

with strict inequality if and only if $I_{k} \subsetneq I_{1} \cup I_{2}$, for each $k=1,2$. Therefore, combining (2) and (3), the result follows.

Corollary 1 Under the hypotheses of Lemma 1, if either $W_{1}$ or $W_{2}$ is full-rank, then $\alpha A W_{1}+$ $(1-\alpha) A W_{2}$ is full-rank for each $\alpha \in(0,1)$.

Remark 2 The result of Lemma 1 can be easily transformed in terms of concavity by simply extending it to the case $\alpha \in[0,1]$. Indeed, using the notation of Lemma 1 , for any $\alpha \in[0,1]$ the rank operator satisfies:

$$
\operatorname{rank}\left(\alpha A W_{1}+(1-\alpha) A W_{2}\right) \geq \min \left\{\operatorname{rank}\left(A W_{1}\right), \operatorname{rank}\left(A W_{2}\right)\right\}
$$

with strict inequality for some value of $\alpha \in(0,1)$ if and only if $I_{k} \subsetneq I_{1} \cup I_{2}, k=1,2$. We conclude that the rank operator is a is a quasi-concave function.

On the base of the previous results, the following proposition highlights important concavity properties of the function $\mathcal{F}_{C}$.

Proposition 2 (Quasi-concavity) Let $C$ be a function of $\mathcal{H}^{m, n}$ which assumes values in $[1,+\infty)$ and satisfying property (vi) of Definition 1 . Then, $\mathcal{F}_{C}$ is a quasi-concave function of $\mathcal{H}^{m, n}$, that is for each nonzero $A \in$ Mat $_{m \times n}, \alpha \in[0,1], \boldsymbol{w}_{1}, \boldsymbol{w}_{2} \in \mathbb{W}_{n}$, the following result holds:

$$
\mathcal{F}_{C}\left(\alpha \boldsymbol{w}_{1}+(1-\alpha) \boldsymbol{w}_{2}, A\right) \geq \min \left\{\mathcal{F}_{C}\left(\boldsymbol{w}_{1}, A\right), \mathcal{F}_{C}\left(\boldsymbol{w}_{2}, A\right)\right\}
$$

with strict inequality for at least one value of $\alpha \in(0,1)$ when $I_{k} \subsetneq I_{1} \cup I_{2}, k=1,2$, where $I_{k}=\left\{j \in\{1, \ldots, n\} \mid w_{k j}>0\right\}$.

Proof Let $W_{1}=\operatorname{diag}\left(\boldsymbol{w}_{1}\right), W_{2}=\operatorname{diag}\left(\boldsymbol{w}_{2}\right) \in \operatorname{Mat}_{n \times n}$. The cases $\alpha=0$ and $\alpha=1$ are trivially verified. Let $\alpha \in(0,1)$ and let $I_{k}=\left\{j \in\{1, \ldots, n\} \mid w_{k j}>0\right\}$, with $k=1,2$. If $I_{k} \subsetneq I_{1} \cup I_{2}$, for each $k=1,2$, then Lemma 1 yields $\operatorname{rank}\left(\alpha A W_{1}+(1-\alpha) A W_{2}\right)>$ $\max \left\{\operatorname{rank}\left(A W_{1}\right), \operatorname{rank}\left(A W_{2}\right)\right\}$, and applying Proposition 1, item 4, it follows:

$$
\mathcal{F}_{C}\left(\alpha \boldsymbol{w}_{1}+(1-\alpha) \boldsymbol{w}_{2}, A\right)>\max \left\{\mathcal{F}_{C}\left(\boldsymbol{w}_{1}, A\right), \mathcal{F}_{C}\left(\boldsymbol{w}_{2}, A\right)\right\}
$$

Otherwise, assume that one of $I_{k}, k=1,2$, is such that $I_{k}=I_{1} \cup I_{2}$. W.l.o.g. we assume that $k=1$, so that $I_{2} \subseteq I_{1}$. Assume that $I_{2} \subsetneq I_{1}$. Lemma 1 yields $\operatorname{rank}\left(\alpha A W_{1}+\right.$ $\left.(1-\alpha) A W_{2}\right)=\operatorname{rank}\left(A W_{1}\right)>\operatorname{rank}\left(A W_{2}\right)$ and applying Proposition 1, item 4 , it follows 
$\mathcal{F}_{C}\left(\boldsymbol{w}_{1}, A\right)>\mathcal{F}_{C}\left(\boldsymbol{w}_{2}, A\right)$. Therefore, letting $W_{\alpha}=\alpha W_{1}+(1-\alpha) W_{2}$ and using Proposition 1 , item 3 , we get

$$
\begin{aligned}
f_{C}\left(\alpha \boldsymbol{w}_{1}+(1-\alpha) \boldsymbol{w}_{2}, A\right) & =\operatorname{rank}\left(A W_{\alpha}\right)+\frac{1}{C\left(A W_{\alpha}\right)}-1 \\
& =\operatorname{rank}\left(A W_{1}\right)+\frac{1}{C\left(A W_{\alpha}\right)}-1 \\
& >\operatorname{rank}\left(A W_{1}\right)-1 \geq \operatorname{rank}\left(A W_{2}\right) \\
& \geq \operatorname{rank}\left(A W_{2}\right)+\frac{1}{C\left(A W_{2}\right)}-1=f_{C}\left(\boldsymbol{w}_{2}, A\right),
\end{aligned}
$$

which yields $\mathcal{F}_{C}\left(\alpha \boldsymbol{w}_{1}+(1-\alpha) \boldsymbol{w}_{2}, A\right) \geq \min \left\{\mathcal{F}_{C}\left(\boldsymbol{w}_{1}, A\right), \mathcal{F}_{C}\left(\boldsymbol{w}_{2}, A\right)\right\}$.

Finally, if $I_{2}=I_{1}$, Lemma 1 assures that $\operatorname{rank}\left(\alpha A W_{1}+(1-\alpha) A W_{2}\right)=\operatorname{rank}\left(A W_{1}\right)=$ $\operatorname{rank}\left(A W_{2}\right)$; exploiting the quasi-convexity of $C$ we get

$$
\begin{aligned}
f_{C}\left(\alpha \boldsymbol{w}_{1}+(1-\alpha) \boldsymbol{w}_{2}, A\right) & =\operatorname{rank}\left(A W_{i}\right)+\frac{1}{C\left(\alpha A W_{1}+(1-\alpha) A W_{2}\right)}-1 \\
& \geq \operatorname{rank}\left(A W_{i}\right)+\frac{1}{\max \left\{C\left(A W_{1}\right), C\left(A W_{2}\right)\right\}}-1 \\
& =\min \left\{f_{C}\left(\boldsymbol{w}_{1}, A\right), f_{C}\left(\boldsymbol{w}_{2}, A\right)\right\},
\end{aligned}
$$

which yields $\mathcal{F}_{C}\left(\alpha \boldsymbol{w}_{1}+(1-\alpha) \boldsymbol{w}_{2}, A\right) \geq \min \left\{\mathcal{F}_{C}\left(\boldsymbol{w}_{1}, A\right), \mathcal{F}_{C}\left(\boldsymbol{w}_{2}, A\right)\right\}$.

In the final part of the section we enumerate the technical results for additional properties on the function $\mathcal{F}_{C}$. We provide the sufficient conditions the connectedness measure $C$ needs to verify in order to define a CPDM through $\mathcal{F}_{C}$. In particular, Proposition 3 proves the Duplication invariance, Proposition 4 the Size monotonicity, Proposition 5 the Translation invariance, Proposition 6 the Homogeneity and Proposition 7 the Symmetry (see also CPDMs axioms, Section 2).

Proposition 3 (Duplication invariance) Let $C$ be a function of $\mathcal{H}^{m, n}$ that satisfies properties (ii), (iii), (iv) of Definition 1. Let $A \in M_{m \times n}$ be a nonzero matrix, $k \in\{1, \ldots, n\}$ and $A^{+}=\left(A \mid A^{k}\right) \in \operatorname{Mat}_{m \times(n+1)}$. Let $\boldsymbol{w} \in \mathbb{W}_{n}$ and $\boldsymbol{w}^{+} \in \mathbb{W}_{n+1}$ be such that $w_{j}^{+}=w_{j}$, for each $j=1, \ldots, n, j \neq k$, and $w_{k}^{+}+w_{n+1}^{+}=w_{k}$. Then $\mathcal{F}_{C}\left(\boldsymbol{w}^{+}, A^{+}\right)=\mathcal{F}_{C}(\boldsymbol{w}, A)$.

Proof Let $W=\operatorname{diag}(\boldsymbol{w}) \in \operatorname{Mat}_{n \times n}$ and $W^{+}=\operatorname{diag}\left(\boldsymbol{w}^{+}\right) \in \operatorname{Mat}_{(n+1) \times(n+1)}$. Recalling Definition 4 , it is enough to prove that $\operatorname{rank}\left(A^{+} W^{+}\right)=\operatorname{rank}(A W)$ and $C\left(A^{+} W^{+}\right)=$ $C(A W)$. The first equality is immediate; we prove the second equality by considering the following three cases: $w_{k}^{+}=w_{k}$ and $w_{n+1}^{+}=0 ; w_{k}^{+}=0$ and $w_{n+1}^{+}=w_{k} ; w_{k}^{+}>0$ and $w_{n+1}^{+}>0$. In the first case, $w_{k}^{+}=w_{k}$ and $w_{n+1}^{+}=0$, the matrix $A^{+} W^{+}$can be expressed as $A^{+} W^{+}=\left(A W \mid \mathbf{0}_{m \times 1}\right)$, so the invariance by size property of $C$ yields $C\left(A^{+} W^{+}\right)=C(A W)$. In the second case, $w_{k}^{+}=0$ and $w_{n+1}^{+}=w_{k}$, the matrix $A^{+} W^{+}$can be expressed as $A^{+} W^{+}=\left(A W \mid \mathbf{0}_{m \times 1}\right) \Pi$, where $\Pi$ is a permutation matrix only exchanging the $k$ th and $(n+1)$ th positions. Applying the invariance by columns permutations and by size we obtain $C\left(A^{+} W^{+}\right)=C(A W)$. In the last case, $w_{k}^{+}>0$ and $w_{n+1}^{+}>0$, with $w_{k}^{+}+w_{n+1}^{+}=w_{k}$, we let $w_{k^{+}}=b w_{k}$, with $b>0$. The matrix $A^{+} W^{+}$can be expressed as

$$
A^{+} W^{+}=\left(A^{1} w_{1}, \ldots, A^{k-1} w_{k-1}, \alpha A^{k} w_{k}, A^{k+1} w_{k+1}, \ldots, A^{n} w_{n}, A^{k} w_{n+1}^{+}\right),
$$

and applying the invariance for size and under one scalar multiplication we obtain $C\left(A^{+} W^{+}\right)=C\left(A^{1} w_{1}, \ldots, c A^{k} w_{k}, \ldots, A^{n} w_{n}\right)=C(A W)$. 
Proposition 4 (Size monotonicity) Let $C$ be a function of $\mathcal{H}^{m, n}$ that assumes values in $[1,+\infty)$ and satisfies properties (ii), (v) of Definition 1. Let $A \in \mathrm{Mat}_{m \times n}$ be a nonzero matrix and $A^{+}=\left(A \mid A^{n+1}\right) \in \operatorname{Mat}_{m \times(n+1)}$ with $A^{n+1} \neq A^{j}$ for each $j=1, \ldots, n$. Let $\boldsymbol{w}^{+} \in \mathbb{W}_{n+1}$ be such that $\overline{\boldsymbol{w}}=\sum_{j=1}^{n} w_{j}>0$ and let $\boldsymbol{w}=\frac{1}{\overline{\boldsymbol{w}}}\left(w_{1}, \ldots, w_{n}\right) \in \mathbb{W}_{n}$. Then $\mathcal{F}_{C}\left(\boldsymbol{w}^{+}, A^{+}\right) \geq \mathcal{F}_{C}(\boldsymbol{w}, A)$.

Proof Let $W=\operatorname{diag}(\boldsymbol{w}) \in \operatorname{Mat}_{n \times n}$ and $W^{+}=\operatorname{diag}\left(\boldsymbol{w}^{+}\right) \in \operatorname{Mat}_{(n+1) \times(n+1)}$. By hypothesis on $\boldsymbol{w}$ and $\boldsymbol{w}^{+}$, the matrix $A^{+} W^{+}$can be expressed as $A^{+} W^{+}=\left(\overline{\boldsymbol{w}} A W \mid w_{n+1} A^{n+1}\right)$; consequently, we have either $\operatorname{rank}\left(A^{+} W^{+}\right)=\operatorname{rank}(A W)+1$ or $\operatorname{rank}\left(A^{+} W^{+}\right)=\operatorname{rank}(A W)$. In the first case, $\operatorname{rank}\left(A^{+} W^{+}\right)=\operatorname{rank}(A W)+1>\operatorname{rank}(A W)$, so Proposition 1 item 4 yields $\mathcal{F}_{C}\left(\boldsymbol{w}^{+}, A^{+}\right)>\mathcal{F}_{C}(\boldsymbol{w}, A)$. In the second case, that is if $\operatorname{rank}\left(A^{+} W^{+}\right)=\operatorname{rank}(A W)$, using the properties of invariance for size and 0-degree homogeneity of $C$, it follows that:

$$
C\left(A^{+} W^{+}\right)=C\left(\left(\overline{\boldsymbol{w}} A W \mid w_{n+1} A^{n+1}\right)\right)=C(\overline{\boldsymbol{w}} A W)=C(A W),
$$

therefore $\mathcal{F}_{C}\left(\boldsymbol{w}^{+}, A^{+}\right)=\mathcal{F}_{C}(\boldsymbol{w}, A)$.

Proposition 5 (Translation invariance) Let $C$ be a function of $\mathcal{H}^{m, n}$ that satisfies property $(i)$ of Definition 1, let $A \in$ Mat $_{m \times n}$ be a nonzero matrix and $\alpha \in \mathbb{R}$. If no rows and no columns of $A$ are equal to $\mathbf{1}_{1 \times n}$ and $\mathbf{1}_{m \times 1}$ respectively, then $\mathcal{F}_{C}(\boldsymbol{w}, A+\alpha)=\mathcal{F}_{C}(\boldsymbol{w}, A)$ for each $\boldsymbol{w} \in \mathbb{W}_{n}$.

Proof Let $W=\operatorname{diag}(\boldsymbol{w}) \in \operatorname{Mat}_{n \times n}$ and note that the matrix $A+\alpha$ can be expressed as $A+\alpha=A+\alpha \mathbf{1}_{m \times n}$, with $\operatorname{rank}\left(\alpha \mathbf{1}_{m \times n}\right)=1$ in the nontrivial case $\alpha \neq 0$. Consequently $(A+\alpha) W=A W+\alpha \mathbf{1}_{m \times n} W$ and, using the hypothesis on $A$ and the rank-sum inequality, see Horn and Johnson [15, Section 0.4.5], we obtain $\operatorname{rank}((A+\alpha) W)=\operatorname{rank}(A W)$. Further, the property of $C$ of being invariant under independent translations yields $C((A+\alpha) W)=$ $C\left(A W+\alpha \mathbf{1}_{m \times n} W\right)=c(A W)$ which, coupled with the rank result and Definition 4 , concludes the proof.

Proposition 6 (Homogeneity) Let $C$ be a function of $\mathcal{H}^{m, n}$ that satisfies property (v) of Definition 1, let $A \in \operatorname{Mat}_{m \times n}$ be a nonzero matrix and $\alpha>0$. Then $\mathcal{F}_{C}(\boldsymbol{w}, \alpha A)=\mathcal{F}_{C}(\boldsymbol{w}, A)$ for each $w \in \mathbb{W}_{n}$.

Proof Let $W=\operatorname{diag}(\boldsymbol{w}) \in \operatorname{Mat}_{n \times n}$. By recalling Definition 4 and exploiting the equality $\operatorname{rank}(\alpha A W)=\operatorname{rank}(A W)$ and the degree 0 homogeneity of $C$, it immediately follows that $\mathcal{F}_{C}(\boldsymbol{w}, \alpha A)=\mathcal{F}_{C}(\boldsymbol{w}, A)$.

Proposition 7 (Symmetry) Let $C$ be a function of $\mathcal{H}^{m, n}$ that satisfies property (iii) of Definition 1, et $A \in \operatorname{Mat}_{m \times n}$ be nonzero and $\Pi \in$ Mat $_{n \times n}$ be a permutation matrix. Then $\mathcal{F}_{C}(\Pi w, A \Pi)=\mathcal{F}_{C}(\boldsymbol{w}, A)$ for each $\boldsymbol{w} \in \mathbb{W}_{n}$.

Proof Let $W=\operatorname{diag}(\boldsymbol{w}), W_{\Pi}=\operatorname{diag}(\Pi \boldsymbol{w}) \in \mathrm{Mat}_{n \times n}$; we have $W_{\Pi}=\Pi^{t} W \Pi$. We consider the matrix $A \Pi W_{\Pi}=A \Pi \Pi^{t} W \Pi=A W \Pi: \operatorname{since} \operatorname{rank}(A W \Pi)=\operatorname{rank}(A W)$ and, by hypothesis on $C, C(A W \Pi)=C(A W)$, recalling Definition 4, the result immediately follows.

\section{Induced portfolio diversification measures}

In this section, starting from four fixed PMCs, the Maximum Variance Inflation Factor (MVIF), see Belsey et al. [2], the Power Mean measures $\mu_{k}^{1}$, see Maggi et al. [18], the Market 
Rank Indicator (MRI), see Figini et al. [12], and the Cumulative Risk Fraction $h_{k}$, see Billio et al. [3], and using the function $\mathcal{F}$ introduced in Sect. 3, see Definition 4, we explicitly construct original portfolio diversification measures. We prove that $\mathcal{F}_{\mathrm{M} \text {-VIF }}$ is a CPDM, whereas $\mathcal{F}_{\mu_{k}^{1}}, \mathcal{F}_{s_{k}}$ and $\mathcal{F}_{h_{k}}$ only partially verify the theoretical requirements of CPDMs. Since, in general, the PMCs are only defined in the case of full-rank matrices, we introduce for each considered PMC a possible generalization that, exploiting the classical GaussJordan elimination method, allows us to treat even the case of rank-deficient matrices. To this end, in the rest of the section, for each nonzero matrix $A \in \mathrm{Mat}_{m \times n}$ we denote by $r=\operatorname{rank}(A-\bar{A})$ and by $(A-\bar{A})^{P}=\left((A-\bar{A})^{p_{1}}, \ldots,(A-\bar{A})^{p_{r}}\right)$ the matrix obtained applying the Gauss-Jordan elimination method to $A-\bar{A}$.

\subsection{The Variance Inflation Factor}

In Maggi et al. [18], based on the classical econometric measure known as Variance Inflation Factors (VIFs), see for instance Belsey et al. [2], it is proved that the maximum VIFs provides a measure of connectedness. In the following definition we recall and extend this notion to be defined for mean-centered variables even in the case of rank deficient matrices (we also refer to Gross [13] for the definition of the centered VIFs).

Definition 5 (Maximum Variance Inflation Factor-M-VIF) The Variance Inflation Factors $\mathrm{VIF}_{j}:\left\{A \in \operatorname{Mat}_{m \times n} \backslash\left\{\mathbf{0}_{m \times n}\right\} \mid r=\operatorname{rank}(A-\bar{A})>1\right\} \rightarrow \mathbb{R}, j=1, \ldots, r$, are defined by

$$
\operatorname{VIF}_{j}(A)=\operatorname{VIF}_{j}\left((A-\bar{A})^{P}\right)=\frac{1}{1-R_{j}^{2}}, \quad j=1,2, \ldots, r,
$$

where $R_{j}^{2}$ is the coefficient of determination of the linear regression of $(A-\bar{A})^{p_{j}}$ with respect to $\left\{(A-\bar{A})^{p_{i}} \mid i=1, \ldots, r, i \neq j\right\}$. The Maximum Variance Inflation Factor M-VIF : Mat ${ }_{m \times n} \backslash\left\{\boldsymbol{0}_{m \times n}\right\} \rightarrow \mathbb{R}$ is defined by

$$
\operatorname{M-VIF}(A)= \begin{cases}1 & \text { if } r=1 \\ \max \left\{\operatorname{VIF}_{1}(A), \ldots, \operatorname{VIF}_{r}(A)\right\} & \text { if } r>1\end{cases}
$$

for each $A \in \operatorname{Mat}_{m \times n} \backslash\left\{\mathbf{0}_{m \times n}\right\}$.

The measure M-VIF verifies the following important properties.

Proposition 8 The measure $M$-VIF assumes values in $[1,+\infty)$ and satisfies properties $(i)-(v i)$ of Definition 1.

Proof Let $A \in \operatorname{Mat}_{m \times n}$ be a nonzero matrix. In the trivial case of $r=1$ then $\operatorname{M-VIF}(A)=1$; otherwise, since each coefficient of determination $R_{j}^{2} \in[0,1]$, it holds $\operatorname{VIF}_{j}(A) \geq 1$ and consequently $\operatorname{M-VIF}(A) \in[1,+\infty)$. We prove $(i)-(v i)$.

(i) We consider the nontrivial case $r>1$ and note that the coefficient of determination $R_{j}$ satisfies $R_{j}^{2}=1-\frac{\left\|r_{j}\right\|^{2}}{\sum_{i=1}^{r}\left\|A^{p_{i}}-\overline{A^{p_{i}}}\right\|^{2}}$ where, for each $i=1, \ldots, r, r_{j}$ is the residual vector of the linear regression of $A^{p_{j}}-\overline{A^{p_{j}}}$ with respect to $\left\{A^{p_{i}}-\overline{A^{p_{i}}} \mid i=1, \ldots, r, i \neq j\right\}$. Then $1-R_{j}^{2}$ is invariant under independent translation of the columns of $A$. In the rest of the proof w.l.o.g. we assume that each $\overline{A^{j}}=0$.

(ii) We let $b \in \operatorname{Mat}_{m \times 1}$ and suppose that $\operatorname{rank}(A \mid b)=\operatorname{rank}(A)=r$. If $r=1$ then by definition $\operatorname{M-VIF}((A \mid b))=\operatorname{M-VIF}(A)=1$. Otherwise, the result follows simply by 
observing that the lists of pivots obtained by the Gauss-Jordan elimination applied to $A$ and $(A \mid b)$ are the same.

(iii) We consider $A \in \mathcal{M}_{m \times n}$ and observe that the property simply follows from axiom $C 2$ of PMC.

(iv) We consider the nontrivial case $r>1$ and note that the coefficient of determination $R_{j}$ satisfies $R_{j}^{2}=1-\frac{\left\|r_{j}\right\|^{2}}{\left\|A^{p_{j}}\right\|^{2}}$. Therefore $\operatorname{VIF}_{j}(A)$ can be expressed as

$$
\operatorname{VIF}_{j}(A)=\frac{\left\|A^{p_{j}}\right\|^{2}}{\left\|r_{j}\right\|^{2}} .
$$

Let $k \in\{1, \ldots, n\}, \alpha>0$ and $\widetilde{A}=\left(A^{1}, \ldots, \alpha A^{k}, \ldots, A^{n}\right)$. The list of pivots obtained by the Gauss-Jordan elimination applied to $\widetilde{A}$ is $P=\left(p_{1}, \ldots, p_{r}\right)$. If $k \neq p_{i}, i=$ $1, \ldots, r$, then the property is trivially verified. Otherwise, if there exists $p_{i} \in P$ such that $p_{i}=k$, we consider each index $j \in\{1, \ldots, r\}$ and distinguish the two cases: $k \neq j$ and $k=j$. In the first case, it is easy to verify that the residual vector of the linear regression of $\widetilde{A}^{p_{j}}=A^{p_{j}}$ with respect to $\left\{A^{p_{1}}, \ldots, \alpha A^{k}, \ldots, A^{p_{r}}\right\} \backslash\left\{A^{p_{j}}\right\}$ is $r_{j}$; consequently, relation (4) yields $\operatorname{VIF}_{j}(\widetilde{A})=\operatorname{VIF}_{j}(A)$. In the second case, the residual vector of the linear regression of $\widetilde{A}^{p_{j}}=\alpha A^{p_{j}}$ with respect to $\left\{A^{p_{1}}, \ldots, A^{p_{r}}\right\} \backslash\left\{A^{p_{j}}\right\}$ is $\alpha r_{j}$; consequently, relation (4) yields $\operatorname{VIF}_{j}(\widetilde{A})=\frac{\left\|\alpha A^{p_{j}}\right\|^{2}}{\left\|\alpha r_{j}\right\|^{2}}=\operatorname{VIF}_{j}(A)$. Then, we have $\mathrm{M}-\mathrm{VIF}(\widetilde{A})=\mathrm{M}-\mathrm{VIF}(A)$ so $\mathrm{M}-\mathrm{VIF}$ is invariant under one scalar multiplication.

(v) By combining item (iv) with Remark 1 we immediately conclude that M-VIF is homogeneous of degree 0 .

(vi) From item (iv) it follows that M-VIF satisfies the property $\operatorname{M-VIF}\left(\alpha A W_{1}+(1-\right.$ $\left.\alpha) A W_{2}\right)=\operatorname{M-VIF}\left(A W_{1}\right)=\operatorname{M-VIF}\left(A W_{2}\right)=\operatorname{M-VIF}(A)$ for each $\alpha \in[0,1]$, $A \in \mathcal{M}_{m \times n}$ and $\boldsymbol{w}_{1}, \boldsymbol{w}_{2} \in \mathbb{W}_{n}$, with $W_{1}=\operatorname{diag}\left(\boldsymbol{w}_{1}\right), W_{2}=\operatorname{diag}\left(\boldsymbol{w}_{2}\right) \in \operatorname{Mat}_{n \times n}$.

Proposition 9 The measure $\mathcal{F}_{M-V I F}$ satisfies axioms D1, D2, D3, D4, D5, D6, D7, D8 and D9 of Definition 3.

Proof The proof deeply exploits the properties of M-VIF shown in Proposition 8. In the following we prove that $\mathcal{F}_{\mathrm{M} \text {-VIF }}$ satisfies the following axioms.

D1. The measure M-VIF is a quasi-convex function assuming values in $[1,+\infty)$. Then, axiom $D 1$ follows by applying Proposition 2 to M-VIF.

D2. We let $A \in \operatorname{Mat}_{m \times n} \backslash\left\{\mathbf{0}_{m \times n}\right\}, r=\operatorname{rank}(A)$ and $E_{j}=\operatorname{diag}\left(\boldsymbol{e}_{j}\right)$, for each $j=1, \ldots, r$. Since $\operatorname{rank}\left(A E_{j}\right)=1$ and $\operatorname{M-VIF}\left(A E_{j}\right)=1$, applying Proposition 1, item 1, we get $\mathcal{F}_{\mathrm{M}-\mathrm{VIF}}\left(\boldsymbol{e}_{j}, A\right)=0$, so that axiom $D 2$ is proved.

D3. We let $A \in \operatorname{Mat}_{m \times n} \backslash\left\{\boldsymbol{0}_{m \times n}\right\}$ be such that $A^{i}=A^{j}$ for each $i, j=1, \ldots, n$. Since $\operatorname{rank}(A W)=1$ and $\operatorname{M-VIF}(A W)=1$ for each $\boldsymbol{w} \in \mathbb{W}_{n}$, with $W=\operatorname{diag}(\boldsymbol{w})$, applying Proposition 1 , item 1 , we get $\mathcal{F}_{\mathrm{M}-\mathrm{VIF}}(\boldsymbol{w}, A)=0$, so that axiom $D 3$ is proved.

$D 4$. The measure M-VIF assumes values in $[1,+\infty)$, therefore axiom $D 4$ follows by applying Proposition 1, item 5, to M-VIF.

D5. The measure M-VIF satisfies properties (ii), (iii), (iv) of Definition 1; so Proposition 3 applied to M-VIF yields that $\mathcal{F}_{\mathrm{M}-\mathrm{VIF}}$ satisfies axiom $D$ 5.

D6. Since the measure M-VIF assumes values in $[1,+\infty)$ and satisfies properties (ii), (v) of Definition 1, using Proposition 4 axiom D6 is proved.

D7. The measure M-VIF satisfies property $(i)$ of Definition 1; then axiom $D 7$ follows from Proposition 5. 
D8. The measure M-VIF satisfies property ( $v$ ) of Definition 1, therefore axiom $D 8$ comes by applying Proposition 6 to M-VIF.

D9. Since the measure M-VIF satisfies property (iii) of Definition 1, axiom D9 is a consequence of Proposition 7.

\subsection{The Power Mean measures $\mu_{k}^{1}$}

The Power Mean measures, see Maggi et al. [18], are a family of proper measures of connectedness defined as the ratio between the greatest singular value of the returns and a specified power mean of its smallest singular values. In the following definition we recall this notion in the special case of arithmetic mean, and extend it to be defined for mean-centered variables even in the case of rank-deficient matrices.

Definition 6 Let $A \in \operatorname{Mat}_{m \times n}$ be a nonzero matrix and $\sigma_{j}\left((A-\bar{A})^{P}\right), j=1, \ldots, r$, be the singular values of $(A-\bar{A})^{P}$. For each $k \in\{1, \ldots, r\}$ the Power Mean measure $\mu_{k}^{1}(A)$ : Mat $_{m \times n} \backslash\left\{\mathbf{0}_{m \times n}\right\} \rightarrow \mathbb{R}$ is defined by:

$$
\mu_{k}^{1}(A)=\frac{\sigma_{1}\left((A-\bar{A})^{P}\right)}{M_{1}\left(\sigma_{r-k+1}\left((A-\bar{A})^{P}\right), \ldots, \sigma_{r}\left((A-\bar{A})^{P}\right)\right)}
$$

for each $A \in \operatorname{Mat}_{m \times n} \backslash\left\{\mathbf{0}_{m \times n}\right\}$, where $M_{1}$ denotes the arithmetic mean.

Proposition 10 The measure $\mu_{k}^{1}$ assumes values in $[1,+\infty)$ and satisfies properties $(i)$, (ii), (iii), (v), (vi) of Definition 1.

Proof Let $A \in$ Mat $_{m \times n}$ be a nonzero matrix and $\sigma_{j}\left((A-\bar{A})^{P}\right)$ be the singular values of $(A-\bar{A})^{P}$. Using the internal property of the arithmetic mean, we get

$$
\begin{aligned}
M_{1}\left(\sigma_{r-k+1}\left((A-\bar{A})^{P}\right), \ldots, \sigma_{r}\left((A-\bar{A})^{P}\right)\right) & \leq \sigma_{r-k+1}\left((A-\bar{A})^{P}\right) \\
& \leq \sigma_{1}\left((A-\bar{A})^{P}\right),
\end{aligned}
$$

which yields $\mu_{k}^{1}(A) \geq 1$, for each $k=1, \ldots, r$. We prove properties (i), (ii), (iii), (v), (vi).

(i) We consider the case that $A$ has no rows and no columns equal to $\mathbf{1}_{1 \times n}$ and $\mathbf{1}_{m \times 1}$ respectively and let $B=\left(b_{i j}\right) \in \mathrm{Mat}_{m \times n}$ such that $b_{i j}=\beta_{j} \in \mathbb{R}$ for each $i=1, \ldots, m$ and $j=1, \ldots, n$. It is straightforward to verify that the mean of $(A+B)^{j}$ is $\bar{A}^{j}+\beta_{j}$ so that, if we denote by $\overline{A+B}$ the matrix of the column means of $A+B$, we have $(A+B)-\overline{A+B}=A-\bar{A}$, therefore the property is verified. In the sequel w.l.o.g. we let each $\overline{A^{j}}=0$.

(ii) By hypothesis $\operatorname{rank}(A \mid b)=\operatorname{rank}(A)=r$, where $b \in$ Mat $_{m \times 1}$; since the Gauss-Jordan elimination of $A$ and $(A \mid b)$ have the same pivots the result follows.

(iii) The property is a consequence of axiom $C 2$ of PMC.

(v) The property follows from the same property on the measure of connectedness $\mu_{k}^{1}$, see Maggi et al. [18].

(vi) We consider $A, B \in \mathcal{M}_{m \times n}$. A result of convex analysis, see for instance Qi and Womersley [21], states that the largest singular value and the sum of the $k$ smallest singular values, viewed as matrix functions, are convex and concave functions respectively. Thus, 
for each $\alpha \in[0,1]$, we get:

$$
\begin{aligned}
\mu_{k}^{1}(\alpha A+(1-\alpha) B) & =\frac{k \sigma_{1}(\alpha A+(1-\alpha) B)}{\sum_{j=r-k+1}^{r} \sigma_{j}(\alpha A+(1-\alpha) B)} \\
& \leq \frac{k\left(\alpha \sigma_{1}(A)+(1-\alpha) \sigma_{1}(B)\right)}{\alpha \sum_{j=r-k+1}^{r} \sigma_{j}(A)+(1-\alpha) \sum_{j=r-k+1}^{k} \sigma_{j}(B)} \\
& =F(\alpha) .
\end{aligned}
$$

It is straightforward to verify that $\max _{\alpha \in[0,1]} F(\alpha)=\max \left\{\mu_{k}^{1}(A), \mu_{k}^{1}(B)\right\}$, which yields the quasi-convexity property $\mu_{k}^{1}(\alpha A+(1-\alpha) B) \leq \max \left\{\mu_{k}^{1}(A), \mu_{k}^{1}(B)\right\}$.

Proposition 11 The measure $\mathcal{F}_{\mu_{k}^{1}}$ satisfies axioms D1, D2, D3, D4, D6, D7, D8 and D9 of Definition 3.

Proof The proof relies on Proposition 10. Here, it is omitted as it is analogous to the case of the measure M-VIF (see proof of Proposition 9).

It is worthwhile to note that the the special case $k=1$ yields the matrix 2-norm condition number $\mathcal{K}$, that is $\mu_{1}^{1} \equiv \mathcal{K}$ (and the same situation occurs with the Market Rank Indicator, see Section 4.3). In this case, the associated portfolio diversification measure is $\mathcal{F}_{\mathcal{K}}(\boldsymbol{w}, A)=$ $1-\left(\operatorname{rank}(A W)+\mathcal{K}(A W)^{-1}-1\right)^{-1}$, which supports the idea that portfolios with highly dependent assets provides less diversification opportunities.

\subsection{The Market Rank Indicator}

The notion of Market Rank Indicator (MRI), see Figini et al. [12], is defined as a ratio of some of the eigenvalues of a given matrix and it is useful to detect the collinearity between the columns of the input matrix. In the following definition we recall the MRI and extend it to be defined for mean-centered variables even in the case of rank-deficient matrices.

Definition 7 Let $A \in \operatorname{Mat}_{m \times n}$ be a nonzero matrix and $\sigma_{j}\left((A-\bar{A})^{P}\right), j=1, \ldots, r$, be the singular values of $(A-\bar{A})^{P}$. For each $k \in\{1, \ldots, r\}$ the Market Rank Indicator $(M R I)$ $s_{k}: \operatorname{Mat}_{m \times n} \backslash\left\{\mathbf{0}_{m \times n}\right\} \rightarrow \mathbb{R}$ is defined by:

$$
s_{k}(A)=\frac{\sigma_{1}\left((A-\bar{A})^{P}\right)}{\left(\prod_{j=k+1}^{r} \sigma_{j}\left((A-\bar{A})^{P}\right)\right)^{\frac{1}{k}}}
$$

for each $A \in \operatorname{Mat}_{m \times n} \backslash\left\{\mathbf{0}_{m \times n}\right\}$.

Proposition 12 The measure $s_{k}$ assumes values in $[1,+\infty)$ and satisfies properties ( $\left.i\right)$, (ii), (iii), (v) of Definition 1.

Proof Let $A \in$ Mat $_{m \times n}$ be a nonzero matrix and $\sigma_{j}\left((A-\bar{A})^{P}\right)$ be the singular values of $(A-\bar{A})^{P}$. Using the internal property of the geometric mean, we get

$$
\left(\prod_{j=r-k+1}^{r} \sigma_{j}\left((A-\bar{A})^{P}\right)\right)^{\frac{1}{k}} \leq \sigma_{r-k+1}\left((A-\bar{A})^{P}\right) \leq \sigma_{1}\left((A-\bar{A})^{P}\right)
$$

which yields $s_{k}(A) \geq 1$, for each $k=1, \ldots, r$. The proof of properties $(i),(i i),(i i i),(v)$ is analogous to the case of $\mu_{k}^{1}$ (see proof of Proposition 10) and therefore is omitted. 
Proposition 13 The measure $\mathcal{F}_{s_{k}}$ satisfies axioms D2, D3, D4, D6, D7, D8 and D9 of Definition 3.

Proof The result exploits Proposition 12 and is obtained arguing analogously to the proof of Proposition 9 to which the reader is referred.

\subsection{The Cumulative Risk Fraction}

The notion of Cumulative Risk Fraction, see Billio et al. [3], is defined as the portion of the variability of the returns explained by the first principal components. In Maggi et al. [18] it is proved that the Cumulative Risk Fraction provides a measure of connectedness. In the following definition we recall this notion, rescale it to assume values in the range $[1,+\infty)$ and extend it to be defined even in the case of rank deficient matrices.

Definition 8 Let $A \in \mathrm{Mat}_{m \times n}$ be a nonzero matrix and $\sigma_{j}\left((A-\bar{A})^{P}\right), j=1, \ldots, r$, be the singular values of $(A-\bar{A})^{P}$. For each $k \in\{1, \ldots, r\}$ the function $h_{k}: \operatorname{Mat}_{m \times n} \backslash\left\{\mathbf{0}_{m \times n}\right\} \rightarrow \mathbb{R}$ is defined by:

$$
h_{k}(A)=\frac{\sum_{j=1}^{r} \sigma_{j}^{2}\left((A-\bar{A})^{P}\right)}{\sum_{j=1}^{k} \sigma_{j}^{2}\left((A-\bar{A})^{P}\right)} .
$$

for each $A \in \operatorname{Mat}_{m \times n} \backslash\left\{\mathbf{0}_{m \times n}\right\}$.

It is easy to observe that, in the case of full-rank matrices, Definition 8 coincides with the inverse of the Cumulative Risk Fraction as given in Billio et al. [3].

Proposition 14 The measure $h_{k}$ assumes values in $[1,+\infty)$ and satisfies properties $(i)$, (ii), (iii), (v) of Definition 1.

Proof Using the definition, it is immediate to verify that $h_{k}(A) \geq 1$, for each $k=1, \ldots, r$. Further, since the Cumulative Risk Fraction is homogeneous of degree 0, see Maggi et al. [18], the same property holds for the measure $h_{k}$. For the proof of properties (i), (ii), (iii), (v) we refer to the analogous case of $\mu_{k}^{1}$ (see proof of Proposition 10).

Proposition 15 The measure $\mathcal{F}_{h_{k}}$ satisfies axioms D2, D3, D4, D6, D7, D8 and D9 of Definition 3.

Proof The proof is based on the results of Proposition 14 and similar to the proof of Proposition 9 , to which the reader is referred.

\section{Conclusions}

In this paper we compared the PMCs and the CPDMs starting from their respective axiomatic characterizations. The generality of the proposed approach permits to discuss the properties a PMC needs to verify in order to belong to the class of CPDMs, once the transformation defined by the function $\mathcal{F}$ is applied. Such transformation function $\mathcal{F}$ supports the intuition described in the introduction that a strong relation links the concepts of connectedness and diversification; in particular, given the number of assets in a portfolio, an increase in the connectedness reflects a reduction of the diversification opportunities. The central result of 
the paper is the definition of the induced CPDM starting from a PMC that verifies some required additional properties. In practice, we showed that the VIF induces a CPDM, while other popular PMCs, as the Cumulative Risk Fraction, the Market Rank Indicator and the Power Mean measures, including the special case of the condition number, only partially verify the requirements to define an induced CPDM. While in the present paper, for the sake of simplicity, we focused on the transformation from connectedness to diversification, in our future research we plan to investigate the opposite transition, from diversification to connectedness.

Conflict of interest The authors declare that they have no conflict of interest.

Funding Open Access funding provided by Università degli Studi di Genova.

Open Access This article is licensed under a Creative Commons Attribution 4.0 International License, which permits use, sharing, adaptation, distribution and reproduction in any medium or format, as long as you give appropriate credit to the original author(s) and the source, provide a link to the Creative Commons licence, and indicate if changes were made. The images or other third party material in this article are included in the article's Creative Commons licence, unless indicated otherwise in a credit line to the material. If material is not included in the article's Creative Commons licence and your intended use is not permitted by statutory regulation or exceeds the permitted use, you will need to obtain permission directly from the copyright holder. To view a copy of this licence, visit http://creativecommons.org/licenses/by/4.0/.

\section{References}

1. Bernardi, S., Leippold, M., Lohre, H.: Maximum diversification strategies along commodity risk factors. Eur. Financ. Manag. 24, 53-78 (2018)

2. Belsley, D.A., Kuh, E., Welsch, R.R.: Regression Diagnostics: Identifying Influential Data and Sources of Collinearity. Wiley, New York (1980)

3. Billio, M., Getmansky, M., Lo, A.W., Pelizzon, L.: Econometric measure of connectedness and systemic risk in the finance and insurance sectors. J. Financ. Econ. 104(3), 535-559 (2012)

4. Bisias, D., Flood, M., Lo, A.W., Valavanis, S.: A survey of systemic risk analytics. Ann. Rev. Financ. Econ. 4, 255-299 (2012)

5. Choueifaty, Y., Coignard, Y.: Toward maximum diversification. J. Portf. Manag. 35, 40-51 (2008)

6. Choueifaty, Y., Froidure, T., Reynier, J.: Properties of the most diversified portfolio. J. Invest. Strat. 2, 49-70 (2013)

7. DeMiguel, V., Garlappi, L., Uppal, R.: Optimal versus Naive diversification: How inefficient is the $1 / \mathrm{N}$ portfolio strategy? Rev. Financ. Stud. 22, 1915-1953 (2009)

8. Diamond, J.M., Abdullah, F.A.: Portfolio asset allocation process: international diversification by country or by sector? Int. J. Comm. Manag. 7, 39-55 (1997)

9. Diebold, F.X., Yilmaz, K.: Measuring financial asset return and volatility spillovers, with application to global equity markets. Econ. J. 119, 158-171 (2009)

10. Diebold, F.X., Yilmaz, K.: Better to give than to receive: predictive directional measurement fo volatility spillovers (with discussion). Int. J. Forecast. 28, 57-66 (2012)

11. Diebold, F.X., Yilmaz, K.: On the network topology of variance decompositions: measuring the connectedness of financial firms. J. Econ. 182, 119-134 (2014)

12. Figini, S., Maggi, M.A., Uberti, P.: The market rank indicator to detect financial distress. Econom. Stat. (2018). https://doi.org/10.1016/j.ecosta.2017.12.001

13. Groß, J.: Linear regression. Lecture notes in statistics. Springer, Berlin, Heidelberg (2003)

14. Hauser, T., Vermeersch, D.: Is country diversification still better than sector diversification? Financ. Markets Portf. Manag. 16(2), 234-253 (2002)

15. Horn, R.A., Johnson, C.R.: Matrix Analysis, 2nd edn. Cambridge University Press, New York (2012)

16. Koumou, G. B., Dionne, G.: Coherent diversification measures in portfolio theory: an axiomatic foundation (August 26, 2016). Available at SSRN: https://ssrn.com/abstract=2830516

17. Lohre, H., Opfer, H., Orszag, G.: Diversifying risk parity. J. Risk 16(5), 53-79 (2014)

18. Maggi, M., Torrente, M., Uberti, P.: Proper measures of connectedness. Ann. Finance 16, 547-571 (2020)

19. Markowitz, H.: Portfolio selection. J. Finance 7, 77-91 (1952) 
20. Meucci, A.: Managing diversification. Risk 22, 74-79 (2009)

21. Qi, L., Womersley, R.S.: On extreme singular values of matrix valued functions. J. Convex Anal. 3(1), 153-166 (1996)

22. Qian, E.: On the financial interpretation of risk contribution: risk budgets do add up. J. Invest. Manag. 4, 1-11 (2006)

23. Roncalli, T., Weisang, G.: Risk parity portfolios with risk factors. Quant. Finance 16, 377-388 (2016)

Publisher's Note Springer Nature remains neutral with regard to jurisdictional claims in published maps and institutional affiliations. 